\title{
AOR
}

Selected Papers of \#AolR2021:

The 22nd Annual Conference of the

Association of Internet Researchers

Virtual Event / 13-16 Oct 2021

\section{DOUYIN AND TIKTOK: A CROSS-LANGUAGE SYSTEMATIC REVIEW OF ACADEMIC SCHOLARSHIP ON SISTER APPS}

\author{
Yu Ting Poh \\ Curtin University \\ Crystal Abidin \\ Curtin University

\section{Introduction}

Following the meteoric rise of TikTok in the global market and the dominance of Douyin (抖音) in the Chinese market, this article maps academic scholarship focusing on Douyin and TikTok. TikTok is the international derivative of Douyin - China's short video platform owned by ByteDance (字节跳动). Short video apps are reshaping content creation and consumption on social media and has attracted scholarly interest. Despite the rapid international interest in TikTok, research often neglects the epistemology of scholarship. We therefore focus our attention on Chinese and English academic publications studying Douyin and TikTok to understand the disciplinary approaches, methodologies and research questions of scholars, and the development of academic scholarship on these two platforms. In doing so, our paper seeks to decenter Anglocentric discourses by developing a joint understanding of Douyin and TikTok scholarship.

Douyin and TikTok are "short video platforms that feature 15 to 60 second videos" (Kaye et al., 2020: 2), comprising user-generated content and professionally-generated content. Launched in 2016, Douyin grew rapidly to cater to a Chinese market which desired social entertainment in a fragmented attention economy. In the global market, ByteDance acquired Musical.ly, a music lip-synching app created by another Chinese tech company, in 2018 and all Musical.ly accounts were migrated to TikTok. Douyin and TikTok share similar features and affordances, although some features (e.g. livestreaming) were unique to Douyin before being introduced to TikTok. We address three key research questions: Firstly, what are the disciplinary approaches and methodologies employed by scholars in studying Douyin and TikTok? Secondly, what are the foci of research questions put forth by scholars? Thirdly, how is the academic scholarship on these two platforms developing independently?

Suggested Citation (APA): Poh, Y. T. \& Abidin, C. (2021, October). Douyin and TikTok: A cross-language systematic review of academic scholarship on sister apps. Paper presented at AolR 2021: The 22nd Annual Conference of the Association of Internet Researchers. Virtual Event: AolR. Retrieved from http://spir.aoir.org. 


\section{Methodology}

This abstract draws upon 104 Chinese and English academic publications published between January 2019 to February 2021. We examine Douyin and TikTok scholarship published in Chinese and English by using the China National Knowledge Infrastructure $(\mathrm{CNKI})$ and Google Scholar. Chinese language publications were searched and collected on CNKI which is currently China's largest integrator with over $95 \%$ of Chinese academic resources. CNKI has an "unassailable market share" as compared to competitors (Chen, 2019). English language publications were searched and collected on Google Scholar due to its "broad reach across interdisciplinary academic scholarship indexed on the Internet and its use in prior literature review studies" (Blachnio et al., 2013; Dhir et al., 2013; Kalampokis et al., 2013 as sighted in Snelson, 2016). Of the 104 publications, 58 were published on Douyin ( 7 English, 51 Chinese), 41 were published on TikTok (40 English, 1 Chinese), and 5 featured analyses on Douyin and TikTok (5 English, 0 Chinese).

\section{Findings}

Despite their similarities, these sister apps should not be reduced to interchangeable entities. We note a conflation of both apps in academic research - studies which claimed to focus on one app had in fact analyzed the sister app (e.g. Chen \& Zhang, 2020 as sighted in Abidin, 2021). However, Douyin and TikTok have different ecologies and features, exist in different markets, and should not be simply seen as carbon copies for Chinese and non-Chinese users.

Regarding disciplinary approaches, publications on Douyin tended to be published by scholars in the humanities and social sciences disciplines $(n=50)$. There were significantly fewer publications in the science and technology disciplines $(n=8)$. Publications on TikTok demonstrated a broader scope of disciplinary leanings with scholars from both the humanities and social sciences $(n=26)$ as well as science and technology disciplines $(n=15)$. For comparative publications, 3 were rooted in the humanities and social sciences disciplines while 2 were rooted in the science and technology disciplines.

Regarding methodological approaches, Douyin publications tended to employ qualitative approaches $(n=49)$, with fewer quantitative $(n=4)$ and mixed $(n=5)$ approaches. The representation of qualitative $(n=23)$, quantitative $(n=12)$ and mixed $(n=6)$ approaches for TikTok publications was more balanced. Qualitative $(n=3)$ and quantitative $(n=2)$ approaches were observed in comparative publications. Data collection methods across publications included digital fieldwork, surveys, interviews, experiments, media texts, and ethnography while data analyses included content, thematic, statistical, network, critical discourse, and descriptive forms of analyses.

Regarding research questions, Douyin publications tended to engage with e-commerce $(n=10)$, development of short form videos $(n=8)$, practices, cultures and communities $(n=8)$, intersections of traditional and new media $(n=7)$, platform logics $(n=5)$, transmission of traditional Chinese culture $(n=5)$ and "playful patriotism" $(n=5)$ with the 
latter being defined as "state-endorsed, but not necessarily orchestrated, light-hearted or amusing media content that promotes an established State ideology" (Chen et al., 2021: 111). TikTok publications tended to engage with platform logics and governance concerns $(n=18)$, concepts of fame, virality and influencers $(n=8)$, (re)presentation of events or information $(n=8)$, participatory online video practices and cultures $(n=4)$ as well as engagement and education $(n=3)$. Comparative publications tended to engage with platform logics and platformization $(n=2)$, expression of cultural differences $(n=1)$, fan engagement strategies $(n=1)$ and factors underlying app usage $(n=1)$.

Finally, academic scholarship on Douyin and TikTok are largely based in independent geographic regions. Among 58 Douyin publications, 53 were authored or co-authored by researchers based in universities in mainland China or Chinese regions. For TikTok publications, 35 out of 41 TikTok publications were authored by researchers based in the global North. In the case of comparative publications $(n=5), 1$ was co-authored by researchers based in a Chinese university and another university outside of China. While the remaining 4 publications were authored by researchers based outside of Chinese universities, these researchers (or at least one researcher for co-authored work) were identified to be of Chinese ethnicity based on their names. Given that the digital interfaces of Douyin are displayed in Chinese, the presence of ethnically Chinese co-authors who can make sense of digital affordances was crucial.

\section{Conclusion}

We have identified the early patterns of scholarship foci and found that studies on Douyin and TikTok largely focus on just one app and also demonstrate a Chinese/nonChinese divide in terms of the authors' nationalities. In this regard, we suggest that scholars could take on a comparative perspective to explore these sister apps jointly.

\section{References}

Abidin, C. (2021). Mapping Internet Celebrity on TikTok: Exploring Attention Economies and Visibility Labours. Cultural Science Journal, 12 (1): 77-103.

Chen, P. \& Zhang, X. (2020). Evaluation and empirical study on the information service quality of TikTok government accounts. Eurasian Journal of Social Sciences, 8 (2), 5369.

Chen, R. (2019). Why it's time to reform China's academic publishing databases. Sixth Tone. Retrieved from:

https://sixthtone.medium.com/why-its-time-to-reform-china-s-academic-publishingdatabases-5c969168d76b

Chen, X., Kaye, D. B. V., \& Zeng, J. (2021). \#PositiveEnergy Douyin: Constructing "playful patriotism" in a Chinese short-video application. Chinese Journal of Communication, 14 (1): 97-117. 
Kaye, D. B. V., Chen, X., \& Zeng, J. (2020). The co-evolution of two Chinese mobile short video apps: Parallel platformization of Douyin and TikTok. Mobile Media \& Communication, $00(0)$ : 1-25.

Snelson, C. L. (2016). Qualitative and mixed methods social media research: A review of the literature. International Journal of Qualitative Methods, 1-15. 